\title{
Reliable Mode Tracking in Gradient-Based Optimization Frameworks with Flutter Constraints
}

\author{
Taylor McDonnell \\ Brigham Young University, taylormcd@byu.edu \\ Andrew Ning \\ Brigham Young University, aning@byu.edu
}

Follow this and additional works at: https://scholarsarchive.byu.edu/facpub

Part of the Mechanical Engineering Commons

\section{Original Publication Citation}

McDonnell, T. and Ning, A., "Reliable Mode Tracking in Gradient-Based Optimization Frameworks with Flutter Constraints," AIAA Aviation Forum, June 2021.

\section{BYU ScholarsArchive Citation}

McDonnell, Taylor and Ning, Andrew, "Reliable Mode Tracking in Gradient-Based Optimization Frameworks with Flutter Constraints" (2021). Faculty Publications. 5371.

https://scholarsarchive.byu.edu/facpub/5371 


\title{
Reliable Mode Tracking in Gradient-Based Optimization Frameworks with Flutter Constraints
}

\author{
Taylor McDonnell* and Andrew Ning ${ }^{\dagger}$ \\ Brigham Young University, Provo, UT, 84602, USA
}

\begin{abstract}
In order to construct mode-specific flutter constraints for use in gradient-based multidisciplinary design optimization frameworks, mode tracking must be used to associate the current iteration's modes with the modes corresponding to each constraint function. Existing mode tracking methods, however, do not provide a method by which to ensure the accuracy of mode associations, making them unsuitable for use in situations where obtaining correct mode associations is critical. To remedy this issue, a new mode tracking method is presented which incorporates backtracking logic in order to maintain an arbitrarily high degree of confidence in mode correlations during gradient-based optimization and/or during aeroelastic analyses. This mode tracking method is then applied to the aeroelastic analysis of a linear two-dimensional aeroelastic system and a nonlinear three-dimensional aeroelastic system. Using this mode tracking method in the context of a gradient-based optimization framework eliminates the need to use constraint aggregation to construct flutter constraints appropriate for gradientbased optimization, allows mode shapes to be prescribed, and allows easily controllable modes to be excluded from flutter constraint formulations.
\end{abstract}

\section{Introduction}

As wing aspect ratio increases, dynamic aeroelastic effects such as flutter become increasingly important design drivers, due to increased structural flexibility. Despite the increasing importance of flutter computations, it is still common practice in industry to delay flutter computations until after the initial detailed aircraft design process, since flutter computations require access to aircraft aerodynamic, stiffness, and mass matrices[1]. Delaying flutter computations can lead to costly and time-consuming redesigns if flutter is found within the flight envelope of a proposed aircraft. In addition to being costly and time consuming, this redesign process often results in the development of aircraft that perform worse than originally anticipated.

One commonly proposed method to consider flutter in the initial detailed aircraft design process is to use multidisciplinary design optimization (MDO) to optimize an aircraft's geometry and structure while constraining flutter. MDO with flutter constraints allows the creation of designs that are aeroelastically tailored to be both dynamically stable and highly efficient. MDO without flutter constraints can lead to highly efficient, but ultimately infeasible designs [2, 3].

Since flutter analyses must be performed repeatedly in the context of a MDO, it is necessary that flutter constraints are constructed in a computationally efficient manner. Frequency domain, rather than time-domain analyses are therefore preferred when constructing flutter constraints. Additionally, since gradient-free optimization is computationally intractable when large numbers of design variables are used[4], flutter constraints should be constructed in a manner appropriate for gradient-based optimization. Since gradient-based optimizers are typically designed to work with $C^{1}$ continuous objective and constraint functions, flutter constraints should be $C^{1}$ continuous.

In order to construct $C^{1}$ continuous flutter constraints, the flutter speed cannot be directly constrained, because the identity of the critical aeroelastic mode may switch between design iterations. This phenomenon is known as mode-switching and results in a $C^{0}$ discontinuity if the new mode is a hump mode, and a $C^{1}$ discontinuity otherwise [5]. Mode-switching can be partially prevented through the use of frequency-separation constraints [6, 7], however this approach imposes artificial constraints which can needlessly decrease the performance of the optimized design and does not prevent hump modes. Another approach is to the constrain the real part of each eigenvalue associated with each aeroelastic mode to lie below a preset bounding curve [8-11]. This mitigates the continuity issues, but creates $N_{r} N_{U}$ constraints where $N_{r}$ is the number of modes and $N_{U}$ is the number of speed increments. To reduce the number of constraints, these $N_{r} N_{U}$ constraints are often aggregated using a constraint aggregation function such as the Kreisselmeier-Steinhauser (KS) function[12-15].

\footnotetext{
*Ph.D. Candidate, Department of Mechanical Engineering; taylormcd@byu.edu. Student AIAA.

${ }^{\dagger}$ Associate Professor, Department of Mechanical Engineering; aning@byu.edu. Associate Fellow AIAA.
} 
Even when the stability of each aeroelastic mode is constrained individually, $C^{0}$ discontinuities are possible if the ordering of the eigenvalues associated with each mode is not consistent across design iterations. These discontinuities result from the misassociation of modes with constraint functions from design iteration to design iteration. For flutter constraints derived from linear flutter analyses, a consistent ordering may be defined by ordering aeroelastic modes based on their corresponding structural modes (assuming the ordering of the structural modes is preserved from iteration to iteration)[9]. For flutter constraints based on nonlinear flutter analyses, the relationship between the aeroelastic and structural modes is often not readily apparent and/or easily derived, therefore flutter constraints for nonlinear aeroelastic systems typically use constraint aggregation to achieve $C^{1}$ continuity [3, 16]. The downside of the constraint-aggregation approach, however, is that it precludes the use of mode-specific flutter constraints, as their use would re-introduce dependencies on the ordering of the eigenvalues back into the flutter constraint formulation.

While in many cases, the constraint-aggregation based approach to constructing flutter constraints is sufficient, there are contexts in which modes cannot be consistently ordered based on their corresponding structural modes and mode-specific flutter constraints are desired. Mode-specific flutter constraint functions may be desired, for example, in coupled rigid-body and aeroelastic mode stability analyses, where constraining the phugoid and spiral stability modes to be stable to the same degree as aeroelastic modes could result in over-constrained designs. A mode-specific flutter constraint may also be desired to increase the stability margin on certain modes deemed particularly critical and/or to constrain a mode to match a prescribed shape. Therefore, there exists a need to be able to construct $C^{1}$ continuous mode-specific flutter constraints without ordering aeroelastic modes based on their corresponding structural modes.

To satisfy this need, we develop a new mode-tracking method that can be employed to accurately track modes across design iterations without relying on an aeroelastic mode's relationship with its corresponding structural mode. A salient feature of this mode tracking method is its ability to correlate modes with an arbitrarily high level of confidence, which allows it to avoid generating false mode associations, unlike many other mode tracking methods. To demonstrate our mode tracking method, we apply it to the aeroelastic analysis of a linear two-dimensional aeroelastic system and a nonlinear three-dimensional aeroelastic system, but employ a process that may be used to track modes across the design iterations of a multidisciplinary design optimization.

\section{Theoretical Basis}

This section is divided into three parts. First, we review various mode tracking methods. Then we propose a new mode tracking method which is designed for use in gradient-based optimization frameworks, but is also applicable to standard aeroelastic analyses. Finally, we present the theory associated with the models we use to test our new mode tracking method.

\section{A. Mode Tracking Methods}

The purpose of mode tracking is to establish a correspondence between two or more point solutions to an eigenproblem that are generated using discrete parameter values. Mode tracking is necessary to establish a correspondence between two or more point solutions to an eigenproblem because of the inherently arbitrary ordering of modes resulting from an eigenanalysis. A common application of mode tracking is to relate aeroelastic modes in aeroelastic analysis to structural modes. Correctly establishing the relationship between a mode and its associated structural mode leads to a greater understanding of the specified mode, which can help to inform the design process. Incorrectly establishing the relationship between a aeroelastic mode and its associated structural mode leads to an incorrect understanding of the mode, which may lead to misguided attempts to improve performance.

A variety of mode tracking methods exist with various degrees of complexity. The simplest method to perform mode-tracking is to do so by hand using the judgment of the analyst. While this method seems straightforward, the process becomes challenging and prone to error when correlating modes with similar damping and frequency characteristics. For this reason automatic mode-tracking methods are useful, even outside of optimization frameworks. The simplest automatic mode tracking method is to correlate modes based on complex eigenvalue similarity. This method, however, is prone to errors when eigenvalues associated with different modes occupy similar locations in the complex plane. A more advanced mode tracking method was proposed by Desmarais and Bennett which uses the shape of the characteristic polynomial and Laguerre iteration to converge from a root of the characteristic polynomial for the current iteration to the closest root of the characteristic polynomial for the next iteration[17]. This mode tracking method replaces the eigenproblem reanalysis, potentially resulting in reduced computational expenses, but will result in incorrect correlations if the closest root of the characteristic polynomial does not correspond to the correct mode. Another mode tracking method was presented by Chen[18]. In this method, eigenvalues and their associated 
derivatives for the current iteration are used to predict eigenvalues for the next iteration. Predicted eigenvalues are then used to sort computed eigenvalues based on complex eigenvalue similarity. The error between the predicted and computed eigenvalues is then used to measure the accuracy of the mode tracking and reduce the step size if more accurate results are requested. Unfortunately, similar to the previous two mode tracking methods, the robustness of this method cannot be guaranteed, even with the correction step, since incorrect mode-associations could result when modes have nearly identical eigenvalues. Van Zyl proposed that mode-tracking can be performed by correlating modes based on the similarity of their shapes as measured by the complex inner product between previous and current (right) eigenvectors (essentially an application of the modal assurance criterion to the problem of mode tracking)[19]. Since this method compares eigenvectors rather than eigenvalues it alleviates the mode association issue which occurs when eigenvalues occupy similar locations in the complex plane, but may still fail to choose a correct association if mode shapes change significantly from one step to the next. The method proposed by Van Zyl was improved upon by Eldred et al. by making use of the mass-orthogonality of left and right eigenvectors [20]. This improved method was named the complex cross-orthogonality check method (C-CORC) and was recently extended to the standard eigenvalue problem and demonstrated on a wide variety of aeroelastic problems by Hang et al[21]. Eldred et al. also proposed the complex higher-order eigenpair perturbation (C-HOEP) algorithm for mode tracking[20]. This mode tracking method replaces the eigenproblem reanalysis and is based on the perturbation expansion of the generalized eigenvalue problem. Eldred found C-HOEP to be more robust than C-CORC, but more complicated to implement than C-CORC and therefore recommended either based on the priorities of the designer.

\section{B. New Mode Tracking Method}

Our new mode tracking method modifies C-CORC to incorporate backtracking logic which reduces proposed step sizes if mode correlations are poor. By using an adaptive step size, our mode tracking method is able to maintain an arbitrarily high degree of confidence in mode correlations throughout an aeroelastic analysis or optimization. This new mode tracking method consists of the following core steps:

\section{Generate mode correlations using C-CORC}

The key idea behind C-CORC is to use the biorthogonality of the left and right eigenvectors to recorrelate modes after a parameter perturbation. Given the solutions to the left and right general eigenproblems $\left(\phi^{H} A=\lambda \phi^{H} B\right.$ and $A \psi=\lambda B \psi)$ at steps $i$ and $i+1$ in an iterative process, we can construct the correlation matrix

$$
C=\Phi_{i}^{H} B_{i+1} \Psi_{i+1}
$$

where $\Phi$ and $\Psi$ are matrices of left and right eigenvectors, respectively. For standard eigenproblems $B=I$ and the correlation matrix reduces to

$$
C=\Phi_{i}^{H} \Psi_{i+1}
$$

Due to the biorthogonality of the left and right eigenvectors, the correlation matrix will be diagonal if the parameters used to define $A$ and $B$ are identical at steps $i$ and $i+1$ (assuming the ordering of the modes is the same at steps $i$ and $i+1$ ). If we instead apply a small perturbation to the parameters at step $i$ to obtain the parameters at step $i+1$, the correlation matrix will be diagonally dominant in magnitude, assuming mode-switching has not occurred between steps $i$ and $i+1$. If the correlation matrix is not diagonally dominant in magnitude, mode-switching has occurred and the modes corresponding to each of the columns in $\Psi_{i+1}$ should be rearranged to create a diagonally dominant matrix (if possible) in order to avoid mode-switching.

In practice, sufficiently large step sizes may yield correlation matrices that cannot be reordered to be diagonally dominant. In this case, the correlation matrix may still be used to find mode correlations on a row by row or column by column basis, but the best correlation for each mode might not be unique. To determine correlations row by row, the largest magnitude in each row of the correlation matrix may be assumed to correspond to the best mode correlation at step $i+1$ for each mode at step $i$. To determine correlations column by column, the largest magnitude in each column of the correlation matrix corresponds to the best mode correlation at step $i$ for each mode at step $i+1$. While in principle the row by row or column by column approach to establishing mode correlations is similar, the results from each may differ. Additionally, since the best mode correlation for each mode might not be unique, either approach may fail to yield a one-to-one relationship between previous and current modes, and therefore fail to track some modes in future iterations.

In order to ensure that modes are tracked throughout all iterations, we suggest that a one-to-one correspondence should always be used between the modes at step $i$ and $i+1$. This means that if the best mode correlation for a mode at 
step $i$ is a mode at step $i+1$ that already has a mode correlation, the next best unassigned mode correlation should be used. This ensures that the number of modes that are tracked does not decrease when the best mode correlation for two (or more) different modes at step $i$ is the same mode at step $i+1$.

$\mathrm{C}$-CORC can also be applied to situations where only a subset of eigenvalues/eigenvectors for a given eigenproblem are calculated for computational efficiency. In this case, the eigenvector matrices $\Phi$ and $\Psi$ will be non-square, but the correlation matrix will still be square, assuming the same number of eigenvalues/eigenvectors are computed at steps $i$ and $i+1$. The only requirement when using only a subset of eigenvalues/eigenvectors is that the eigenvalues/eigenvectors of interest are computed at steps $i$ and $i+1$ so that proper mode correlations can be established. Often, more eigenvalues/eigenvectors must be computed than one is actually interested in, in order to ensure that the eigenvalues/eigenvectors of interest are included in the computed subset of eigenvalues/eigenvectors.

\section{Calculate the corruption index for each mode correlation}

The assurance with which a given mode correlation is correct may be measured in C-CORC by using the corruption index, defined as the second largest magnitude in a given row/column of the correlation matrix divided by the largest magnitude in the same row/column (i.e. the best mode correlation excluding the chosen mode correlation divided by the chosen mode correlation). Using this definition, the corruption index varies between zero and one, with values close to zero indicating high confidence in the chosen mode association and values close to one indicating low confidence in the chosen mode association. It may therefore be interpreted as the likelihood of an incorrect mode association. As the magnitude of the parameter perturbations between step $i$ and $i+1$ decrease, the corruption index approaches zero due to the biorthogonality of the left and right eigenvectors. As a result, the corruption index can always be reduced to an arbitrary level by reducing the magnitude of the parameter perturbations, assuming a one-to-one correspondence between previous and current modes exists.

Special consideration must be taken for situations in which multiple correct mode associations exist. Such situations occur, for example, at the point during a parameter sweep where two real eigenvalues transition into a complex conjugate pair of eigenvalues and/or vice versa. If we force a one to one correspondence as suggested previously, modes will still be tracked correctly, but the corruption index will approach one rather than zero as the step size is reduced. In order to ensure that the corruption index decreases as the step size decreases in these scenarios, we redefine the corruption index as the magnitude of the correlation matrix entry corresponding to the $k+1$ best mode correlation divided by magnitude of the correlation matrix entry corresponding to chosen mode correlation where $k$ is the number of entries in the given row or column of the chosen correlation matrix entry that are approximately equal in magnitude to the correlation matrix entry of the chosen mode correlation.

\section{Reduce the step size if any corruption index is too high}

Once proposed mode correlations have been established and the corruption index has been calculated for each mode correlation, we can use this information to determine whether the step size should be reduced in order to improve the quality of the mode-correlations. This reduction in step size helps maintain mode-correlations even when small parameter changes result in large changes in the mode shapes. A lower corruption index tolerance will decrease the likelihood of an incorrect mode association, but increase computational costs. A higher corruption index tolerance will have the opposite effect.

Since backtracking is an essential part of many gradient-based optimizers, this mode tracking technique may be easily implemented with many existing gradient-based optimizers without any modifications. For example, for the optimizer SNOPT, one may trigger backtracking by passing a flag indicating that constraint functions are undefined. For the MATLAB ${ }^{\circledR}$ function fmincon, one may trigger backtracking by returning one or more NaNs from the corresponding constraint functions when mode-correlations fail.

\section{Models}

To test our mode tracking method, we use a linear two-dimensional model constructed by coupling an unsteady aerodynamic model based on Wagner's function with the typical section model and a nonlinear three-dimensional model constructed by coupling Peter's finite state unsteady aerodynamics model with a geometrically exact beam theory model. We first present the theory underlying each model and then present a general method by which any number of models may be coupled together into a single model. 


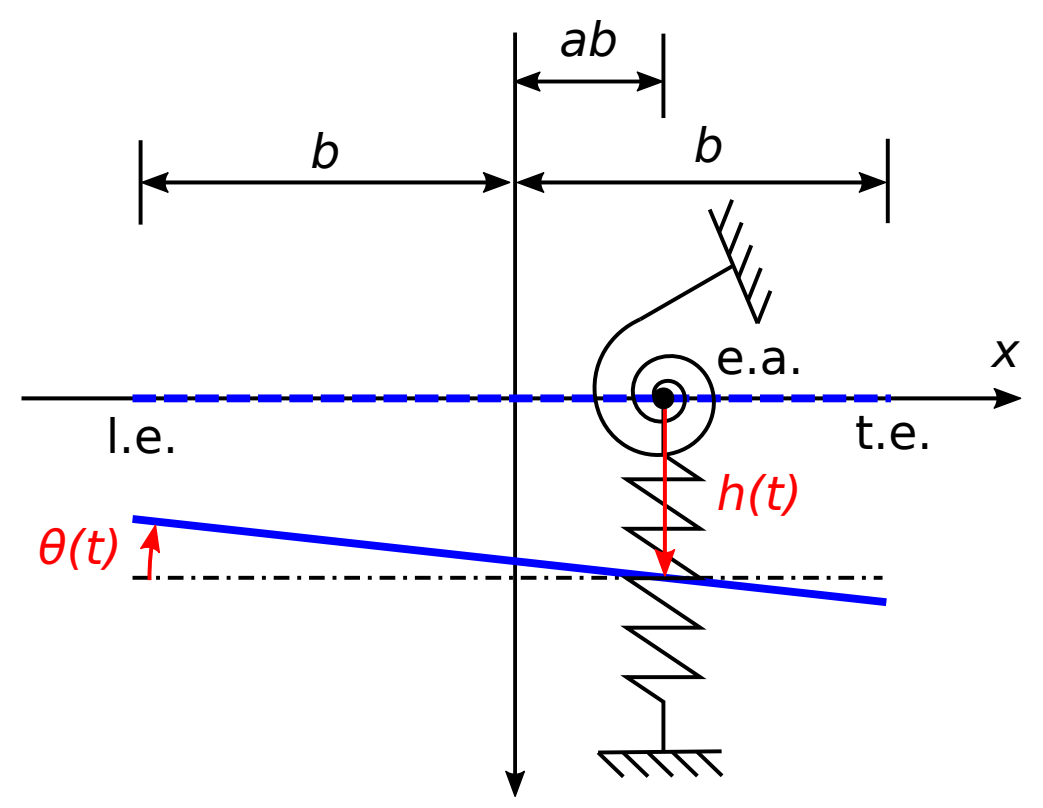

Fig. 1 Classic two degree of freedom typical section model

\section{Typical Section Model}

The equations of motion for the classic typical section model shown in fig. 1 are

$$
\begin{aligned}
m\left(\ddot{h}+b x_{\theta} \ddot{\theta}\right)+k_{h} h & =-\mathcal{L} \\
I_{P} \ddot{\theta}+m b x_{\theta} \ddot{h}+k_{\theta} & =\mathcal{M}
\end{aligned}
$$

where $k_{h}$ is the linear spring constant, $k_{\theta}$ is the torsional spring constant, $m$ is the mass per unit span, $b$ is the semi-chord, $x_{\theta}$ is the x-displacement from the reference point to the center of mass, $I_{P}$ is the moment of inertia about the reference point, $\mathcal{L}$ is the lift per unit span, and $\mathcal{M}$ is the moment about the reference point.

\section{Quasi-Steady Model}

This model is a quasi-steady aerodynamic model derived from thin airfoil theory with inputs and outputs defined as shown in fig. 2. While we don't directly use this model for testing our mode tracking method directly, it forms the foundation for the two other aerodynamic models we use in this paper. The equations for the normal force and moment per unit span at the reference location for this model are:

$$
\begin{aligned}
& \mathcal{N}=a_{0} \rho_{\infty} u^{2} b \alpha_{\mathrm{eff}}+\pi \rho b^{2}(\dot{v}+u \omega-a b \dot{\omega}) \\
& \mathcal{M}=-\pi \rho_{\infty} b^{3}\left[\frac{1}{2} \dot{v}+u \omega+b\left(\frac{1}{8}-\frac{a}{2}\right) \dot{\omega}\right]+b\left(\frac{1}{2}+a\right) \mathcal{N}
\end{aligned}
$$

where $a$ is the normalized distance from the semi-chord to the reference point, $b$ is the semichord length, $a_{0}$ is the lift curve slope, $\rho_{\infty}$ is the air density, $u$ is the local freestream velocity in the chordwise direction, $v$ is the local freestream velocity in the normal direction, $\omega$ is the local freestream angular velocity, and $\alpha_{\text {eff }}$ is the effective angle of attack. The effective angle of attack $\alpha_{\text {eff }}$ for this model is given by

$$
\alpha_{\mathrm{eff}}=\frac{v}{u}+b\left(\frac{1}{2}-a\right) \frac{\omega}{u}-\alpha_{0}
$$

where $\alpha_{0}$ is the zero lift angle of attack. When coupled with the typical section model, the local freestream velocity components are

$$
\begin{aligned}
u & =U_{\infty} \\
v & =U_{\infty} \theta+\dot{h} \\
\omega & =\dot{\theta}
\end{aligned}
$$




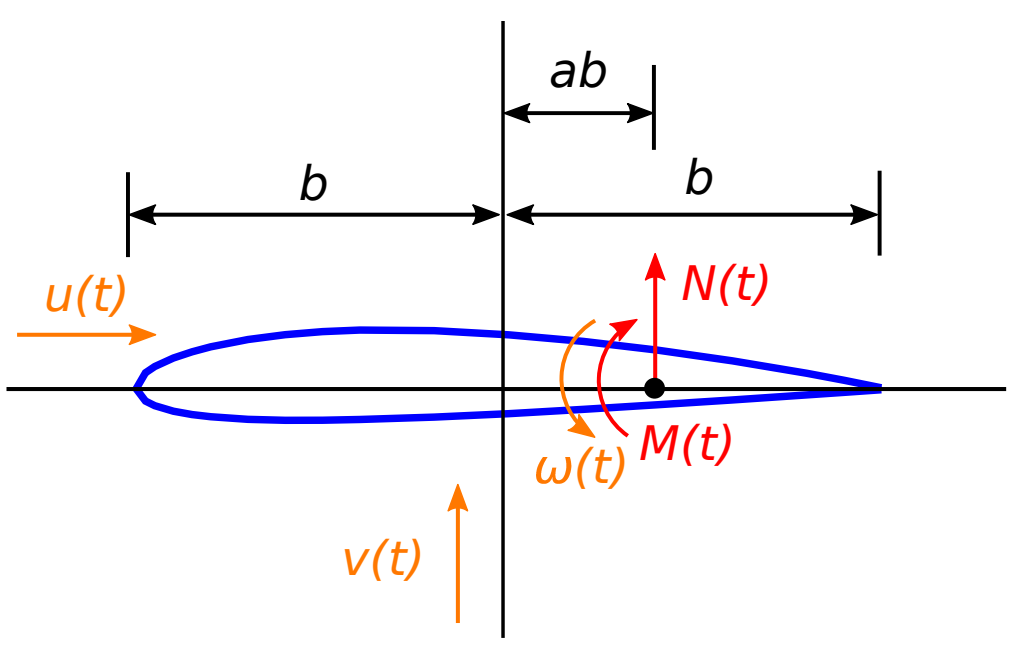

Fig. 2 Inputs and outputs for two-dimensional aerodynamic models

where $U_{\infty}$ is the freestream velocity.

\section{Wagner's Function Model}

Wagner's function models the indicial response of aerodynamic loads under a sudden change in downwash $w$ at the three-quarter chord. The exact expression for Wagner's function is

$$
\phi(t)=\frac{2}{\pi} \int_{0}^{\infty} \frac{\mathfrak{R}(C(\omega)) \sin (\omega(u / b) t)}{\omega} d \omega
$$

where $C(\omega)$ is Theodorsen's function. In many cases, approximate versions of Wagner's function are used rather than the exact expression, of which one of the most common is the approximation of Wagner's function provided by Jones[22]

$$
\phi(t)=1-C_{1} e^{-\varepsilon_{1}(u / b) t}-C_{2} e^{-\varepsilon_{2}(u / b) t}
$$

where $C_{1}=0.165, C_{2}=0.335, \varepsilon_{1}=0.455$, and $\varepsilon_{2}=0.3$.

Wagner's function may be used to model arbitrary airfoil motion using Duhamel's integral. We start by modeling the increment in the circulatory force $\delta \mathcal{N}_{c}(t)$ at time $t$ due to an increment in downwash $\delta w(t)$ at earlier time $\tau$ as

$$
\frac{\delta \mathcal{N}_{c}(t)}{a_{0} \rho_{\infty} u b}=\phi(t-\tau) \delta w(\tau)
$$

where $\phi(t)$ is the impulse response function, which in this case is R. T. Jones' approximation of Wagner's function. Superimposing all previous impulse responses using Duhamel's integral yields the following expression for the circulatory force.

$$
\frac{\mathcal{N}_{c}}{a_{0} \rho_{\infty} u b}=\int_{-\infty}^{t} \frac{\partial w(\tau)}{\partial \tau} \phi(t-\tau) d \tau=w(0) \phi(t)+\int_{0}^{t} \frac{\partial w(\tau)}{\partial \tau} \phi(t-\tau) d \tau
$$

We can transform this equation using integration by parts, yielding

$$
\frac{\mathcal{N}_{c}}{a_{0} \rho_{\infty} u b}=w(t) \phi(0)-\int_{0}^{t} w(\tau) \frac{\partial \phi(t-\tau)}{\partial \tau} d \tau
$$

The integral in this expression may be expressed as a function of the aerodynamic states $\lambda_{1}$ and $\lambda_{2}$.

$$
\begin{aligned}
& \lambda_{1}=C_{1} \varepsilon_{1} \frac{u}{b} \int_{0}^{t} w(\tau) e^{-\varepsilon_{1}(u / b)(t-\tau)} d \tau \\
& \lambda_{2}=C_{2} \varepsilon_{2} \frac{u}{b} \int_{0}^{t} w(\tau) e^{-\varepsilon_{2}(u / b)(t-\tau)} d \tau
\end{aligned}
$$




$$
\lambda_{1}+\lambda_{2}=-\int_{0}^{t} w(\tau) d \phi(t-\tau) d \tau
$$

The expression for circulatory force then reduces to

$$
\frac{\mathcal{N}_{c}}{a_{0} \rho_{\infty} u b}=w(t) \phi(0)+\lambda_{1}+\lambda_{2}
$$

where the downwash at the three-quarter chord is given by

$$
w(t)=v+b\left(\frac{1}{2}-a\right) \omega-u \alpha_{0}
$$

and the aerodynamic state variables $\lambda_{1}$ and $\lambda_{2}$ are described by the ordinary differential equations

$$
\begin{aligned}
& \dot{\lambda_{1}}=-\varepsilon_{1} \frac{u}{b} \lambda_{1}+C_{1} \varepsilon_{1} \frac{u}{b} w(t) \\
& \dot{\lambda_{2}}=-\varepsilon_{2} \frac{u}{b} \lambda_{2}+C_{2} \varepsilon_{2} \frac{u}{b} w(t)
\end{aligned}
$$

The same lift and moment expressions are used as in the quasisteady model, but with a new effective angle of attack

$$
\alpha_{\mathrm{eff}}=\left(\frac{v}{u}+b\left(\frac{1}{2}-a\right) \frac{\omega}{u}-\alpha_{0}\right) \phi(0)+\frac{\lambda_{1}}{u}+\frac{\lambda_{2}}{u}
$$

\section{Peters Finite State Model}

For Peter's finite state model, an additional term is added to the expression for the effective angle of attack from the quasi-steady model to account for the induced velocity from the wake.

$$
\alpha_{\mathrm{eff}}=\frac{v}{u}+b\left(\frac{1}{2}-a\right) \frac{\omega}{u}-\alpha_{0}+\frac{\lambda_{0}}{u}
$$

The induced velocity $\lambda_{0}$ is approximated from a set of $N$ induced-flow states $\lambda_{1}, \lambda_{2}, \ldots, \lambda_{N}$ as

$$
\lambda \approx \frac{1}{2} \sum_{n=1}^{N} b_{n} \lambda_{n}
$$

The set of $\mathrm{N}$ first-order ordinary differential equations which govern the $\mathrm{N}$ finite aerodynamic states are derived by Peters et al.[23] as

$$
\bar{A} \lambda+\frac{u}{b} \lambda=\bar{c}\left[\dot{v}+u \omega+b\left(\frac{1}{2}-a\right) \dot{\omega}\right]
$$

where

$$
\begin{gathered}
\bar{A}=\bar{D}+\bar{d} \bar{b}^{T}+\bar{c} \bar{d}^{T}+\frac{1}{2} \bar{c} \bar{b}^{T} \\
\bar{D}_{n m}=\left\{\begin{array}{ll}
\frac{1}{2 n} & n=m+1 \\
\frac{-1}{2 n} & n=m-1 \\
0 & n \neq m \pm 1
\end{array} \quad \bar{b}_{n}=\left\{\begin{array}{ll}
(-1)^{n-1} \frac{(N+n-1) !}{(N-n-1) !} \frac{1}{(n !)^{2}} & n \neq N \\
(-1)^{n-1} & n=N
\end{array} \quad \bar{c}_{n}=\frac{2}{n} \quad \bar{d}_{n}= \begin{cases}\frac{1}{2} & n=1 \\
0 & n \neq 1\end{cases} \right.\right.
\end{gathered}
$$

\section{Geometrically Exact Beam Theory Model}

This model is a beam model designed to analyze highly-flexible slender structures made of composites. We developed this model based on the mixed formulation of geometrically exact beam theory presented by Wang and Yu[24, 25] and have made it freely available at https://github.com/byuflowlab/GXBeam. $j 1$. As it is a geometrically exact beam model, it is capable of capturing all geometric nonlinearities due to large deflections and rotations, subject to the restriction that strains are small. Applied loads or deflections can be applied at any number of key points, and distributed loads may be applied on each beam element. The governing equations for this model form a set of differential algebraic equations which may be solved using any standard differential algebraic equation solver. 


\section{Lifting Line Model}

Two-dimensional aerodynamic models may be applied in the context of a three-dimensional analysis by applying these models at multiple sections along the span of one or more lifting surfaces. This type of model is applicable when spanwise flow effects are negligible, which is often the case for high aspect ratio wings. For this paper, we use this model in order to extend Peter's finite state model to three dimensions so that it can be coupled with the geometrically exact beam theory model.

The lifting line model we implemented for this paper assumes that the aerodynamics of each section is independent of the aerodynamics of the other sections, except as coupled through structural models. The governing equations for this model are therefore simply the governing equations for each cross-section, concatenated. When coupled with a structural model, local freestream velocities/accelerations are obtained by combining global freestream velocities/accelerations with the velocities/accelerations of the structure at each lifting section. These velocities/accelerations may then be transformed into the local frame of reference using an appropriate transformation matrix. Local aerodynamic forces/moments may then be calculated using a two-dimensional aerodynamic model and transformed back into the reference frame used by the structural model.

\section{Coupling Models}

The governing equations for any number of first order differential equations may be combined by introducing a set of coupling variables $y_{i}$ to the governing differential equations for each model.

$$
\dot{x_{i}}=f_{i}\left(x_{i}, y_{i}, t\right)
$$

Assuming the coupling variables for each model may be defined as functions of the state variables of any/all of the coupled models, the following governing equations may be derived for the coupled system.

$$
\dot{x}=f(x, t)
$$

where

$$
f(x, t)=\left[\begin{array}{c}
f_{1}\left(x_{1}, y_{1}, t\right) \\
f_{2}\left(x_{2}, y_{2}, t\right) \\
\vdots \\
f_{n}\left(x_{n}, y_{n}, t\right)
\end{array}\right] \quad \begin{gathered}
y_{1}=g_{1}(x, t) \\
y_{2}=g_{2}(x, t) \\
\vdots \\
y_{n}=g_{n}(x, t)
\end{gathered} \quad x=\left[\begin{array}{c}
x_{1} \\
x_{2} \\
\vdots \\
x_{n}
\end{array}\right]
$$

Let us now suppose that the governing equations for the any/all of the models is an ordinary differential equation or differential algebraic equation in mass matrix form

$$
M_{i}\left(x_{i}, y_{i}, t\right) \dot{x_{i}}=f_{i}\left(x_{i}, y_{i}, t\right)
$$

The coupled system of equations are then

$$
M(x, t) \dot{x}=f(x, t)
$$

where

$$
\begin{gathered}
M(x, t)=\left[\begin{array}{cccc}
M_{1}\left(x_{1}, y_{1}, t\right) & 0 & 0 & 0 \\
0 & M_{2}\left(x_{2}, y_{2}, t\right) & 0 & 0 \\
0 & 0 & \ddots & 0 \\
0 & 0 & 0 & M_{n}\left(x_{n}, y_{n}, t\right)
\end{array}\right] \\
f(x, t)=\left[\begin{array}{c}
f_{1}\left(x_{1}, y_{1}, t\right) \\
f_{2}\left(x_{2}, y_{2}, t\right) \\
\vdots \\
f_{n}\left(x_{n}, y_{n}, t\right)
\end{array}\right] \quad \begin{array}{c}
y_{1}=g_{1}(x, t) \\
y_{n}=g_{n}(x, t)
\end{array} \quad x=\left[\begin{array}{c}
x_{1} \\
x_{2} \\
\vdots \\
x_{n}
\end{array}\right]
\end{gathered}
$$

If we now add a linearly dependency on the state rates to the definition of the coupling variables, the coupled system of equations become

$$
M(x, t) \dot{x}=f(x, t)
$$


where

$$
\begin{aligned}
& M(x, t)=\left[\begin{array}{cccc}
M_{1}\left(x_{1}, y_{1}, t\right) & 0 & 0 & 0 \\
0 & M_{2}\left(x_{2}, y_{2}, t\right) & 0 & 0 \\
0 & 0 & \ddots & 0 \\
0 & 0 & 0 & M_{n}\left(x_{n}, y_{n}, t\right)
\end{array}\right]+\left[\begin{array}{c}
\frac{\partial f_{1}}{\partial y_{1}} \\
\frac{\partial f_{2}}{\partial y_{2}} \\
\vdots \\
\frac{\partial f_{n}}{\partial y_{n}}
\end{array}\right]\left[\begin{array}{llll}
M_{y_{1}}(x, t) & M_{y_{2}}(x, t) & \ldots & M_{y_{n}}(x, t)
\end{array}\right] \\
& f(x, t)=\left[\begin{array}{c}
f_{1}\left(x_{1}, y_{1}, t\right) \\
f_{2}\left(x_{2}, y_{2}, t\right) \\
\vdots \\
f_{n}\left(x_{n}, y_{n}, t\right)
\end{array}\right] \quad \begin{array}{c}
y_{1}=g_{1}(x, t)-M_{y_{1}}(x, t) \dot{x} \\
y_{2}=g_{2}(x, t)-M_{y_{2}}(x, t) \dot{x} \\
\vdots \\
y_{n}=g_{n}(x, t)-M_{y_{n}}(x, t) \dot{x}
\end{array} \quad x=\left[\begin{array}{c}
x_{1} \\
x_{2} \\
\vdots \\
x_{n}
\end{array}\right]
\end{aligned}
$$

Note that in deriving this final system of equations we have assumed that the state rates for each model are linearly dependent on the coupling parameters, at least for the coupling parameters whose values are dependent on the state rates. This condition must be satisfied in order for the definitions of the mass matrix in eq. (30) to be strictly correct.

\section{Numerical Studies}

In this section, we perform several aeroelastic analyses in order to demonstrate the effectiveness of our mode tracking method. The governing equations for the aeroelastic systems we use to perform these analyses may all be expressed as

$$
M(x) \dot{x}=f(x)
$$

where $M(x)$ is the system mass matrix, which is defined as a function of the state variables, $f(x)$ is a vector-valued function of the state variables which returns the mass matrix multiplied state rates, and $x$ is a vector of state variables. Linearizing this equation about steady state operating conditions $x_{s s}$ produces the following system of equations.

$$
A \tilde{x}=B \dot{\tilde{x}}
$$

where

$$
\tilde{x}=x-x_{s s} \quad A=\left.\frac{\partial f}{\partial x}\right|_{s s} B=M\left(x_{s s}\right)
$$

The eigenvalues and eigenvectors corresponding to the linearized system may be found by solving the generalized eigenvalue problem.

$$
A v=\lambda B v
$$

\section{A. Linear 2D Aeroelastic Analyses}

The first system upon which we test our mode tracking technique is the linear two-dimensional aeroelastic system constructed by coupling the unsteady aerodynamics model based on Wagner's function with the typical section model. To be able to apply the general coupling approach described in this paper, the typical section model is expressed as a first order differential equation with state variables $h, \theta, \dot{h}, \dot{\theta}$ and coupling variables $\mathcal{L}, \mathcal{M}$. For the unsteady aerodynamics model based on Wagner's function, we chose to use the freestream parameters $u, v$, and $\omega$ as coupling parameters, which may be defined as functions of the structural state variables as expressed in eq. (6).

We perform the aeroelastic analysis over a reduced velocity range from 0 to 2 and use the non-dimensional parameters

$$
\begin{array}{cl}
a=-0.3 \quad \mu & =\frac{m}{\rho \pi b^{2}}=10 \quad x_{\theta}=0.05 \\
r_{\theta}^{2}=\frac{I_{\theta}}{m b^{2}} & =0.06 \quad \sigma=\frac{\omega_{h}}{\omega_{\theta}}=0.5 \\
a_{0} & =2 \pi \quad \alpha_{0}=0
\end{array}
$$

The resulting normalized frequency and damping, without any eigenvalue sorting applied, is plotted in fig. 3. Some mode switching may be observed due to the inherently arbitrary ordering of the eigenvalues. 

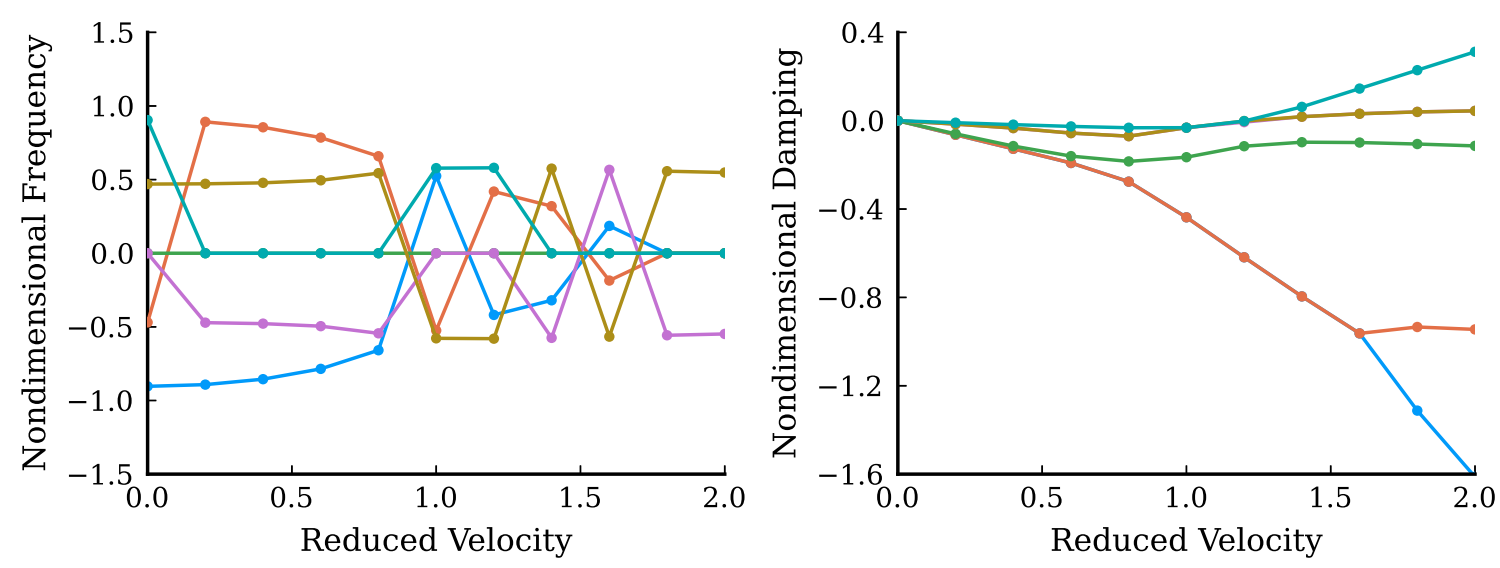

Fig. 3 Frequency and damping of the 2D aeroelastic system without sorting
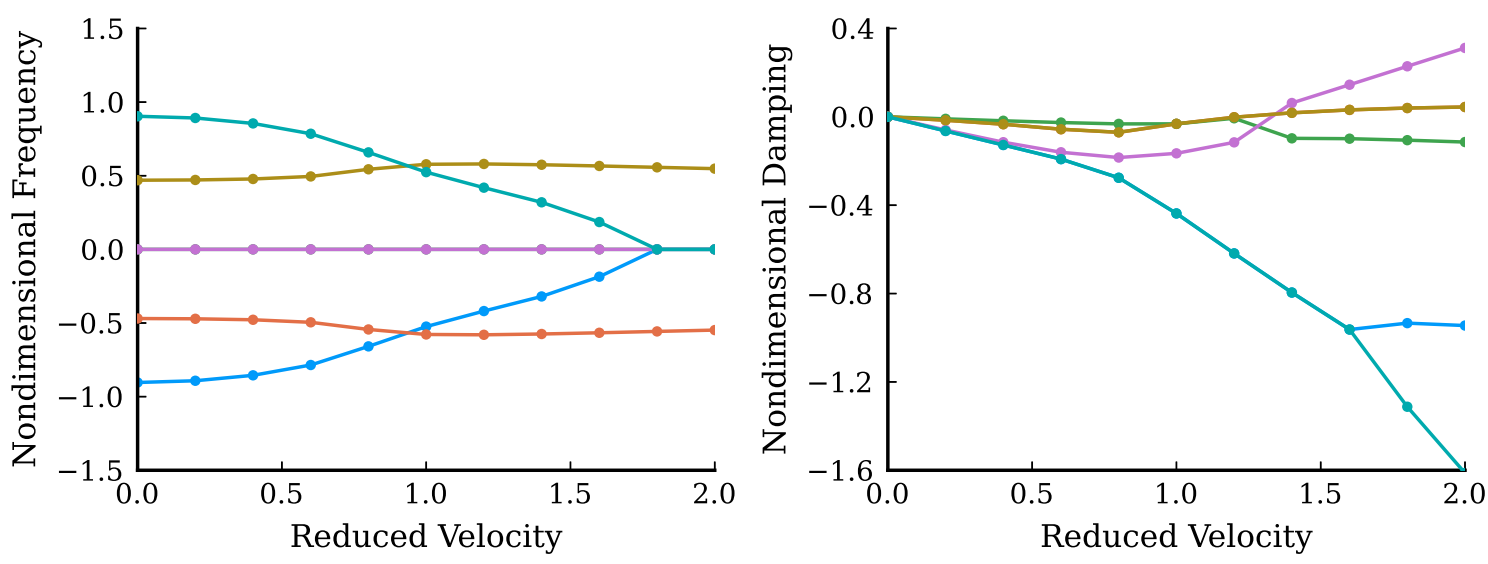

Fig. 4 Frequency and damping of the 2D aeroelastic system with sorting using C-CORC

If we apply C-CORC to track modes as velocity is increased, occurrences of mode switching are greatly reduced, as shown in fig. 4. A false mode association, however, appears to present for the velocity increment from 1.2 to 1.4, since the damping of multiple modes appear to drastically change their trajectories over the corresponding velocity increment. With a sufficiently small velocity increment, all occurrences of mode switching may be eliminated, so we can test whether this mode association is correct or not by using a smaller step size. Unfortunately, the exact step size necessary to eliminate mode switching is problem dependent.

The corruption index associated with each mode association in fig. 4 is shown in fig. 5. Note that in order to ensure that multiple tracked modes don't coalesce into a single tracked mode when mode tracking is applied, we assigned each mode their best unassigned mode correlation rather than allowing modes to be correlated with their best correlated mode. Corruption indices greater than one therefore indicate that two modes shared the best mode correlation, so one of them had to be assigned its next best correlated mode. In this case, two modes share the best same mode correlation for the velocity increment from 1.2 to 1.4 resulting in a corruption index with a magnitude greater than one. Regardless, the high corruption index for the velocity increment from 1.2 to 1.4 indicates that a false mode association likely occurred during that velocity increment, which agrees with our assessment based on our inspection of fig.4.

Figure 6 shows the result of applying the new mode tracking technique presented in this paper, with a corruption index tolerance of 0.5 and simple backtracking logic that halves the step size if the corruption index tolerance is exceeded. As may be observed, the suspected occurrence of mode switching has been eliminated. Additionally, the smooth behavior of the frequency and damping plots suggest that no additional cases of mode switching are present. As might be expected, additional refinement has been added to the analysis at the reduced velocities for which mode switching is likely to occur, which for this case is near the frequency crossing and the transition from complex to real eigenvalues. 


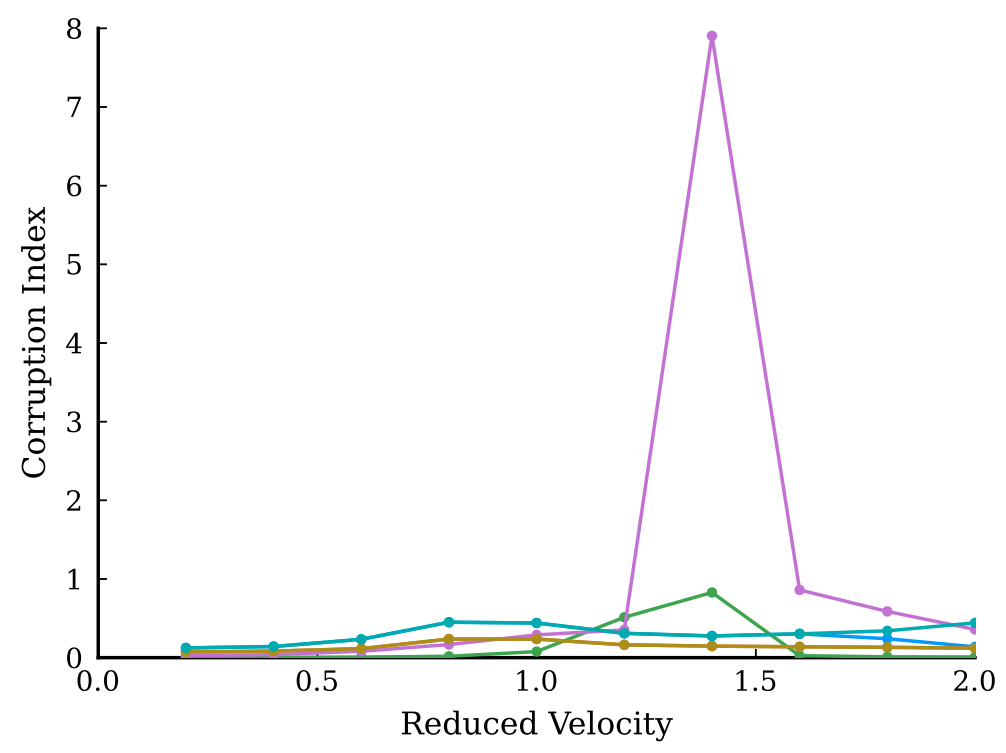

Fig. 5 C-CORC corruption indices for each tracked aeroelastic mode of the 2D aeroelastic system
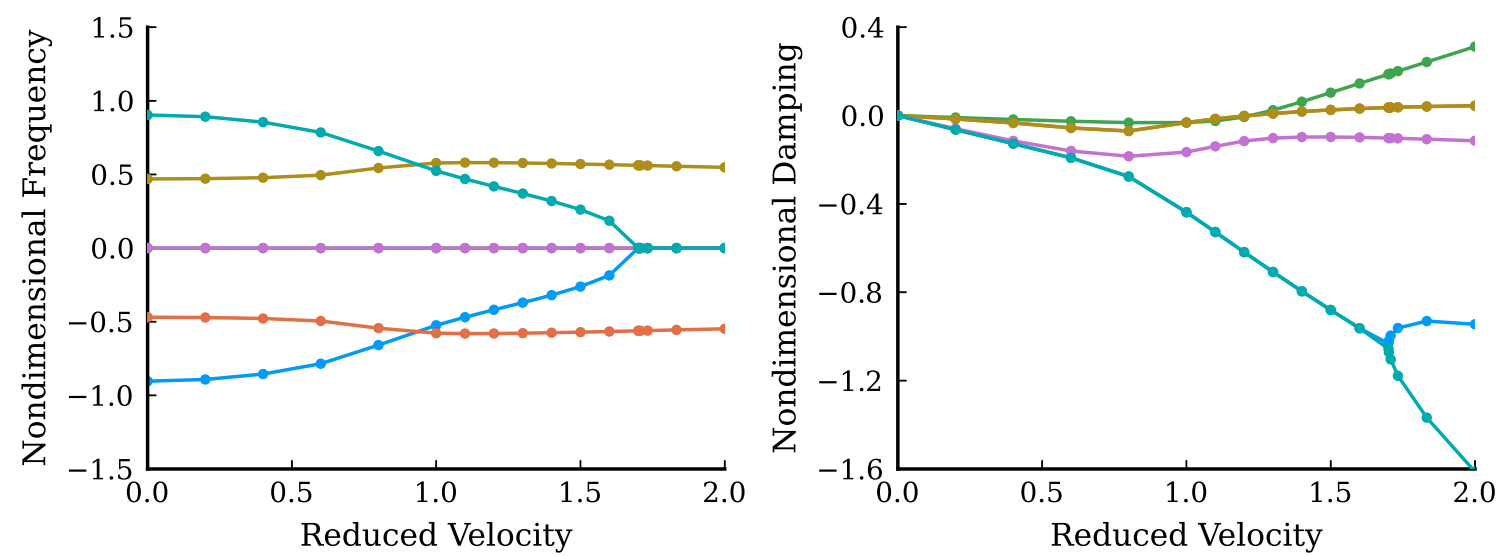

Fig. 6 Frequency and damping of the 2D aeroelastic system with sorting using the new mode tracking method presented in this paper 


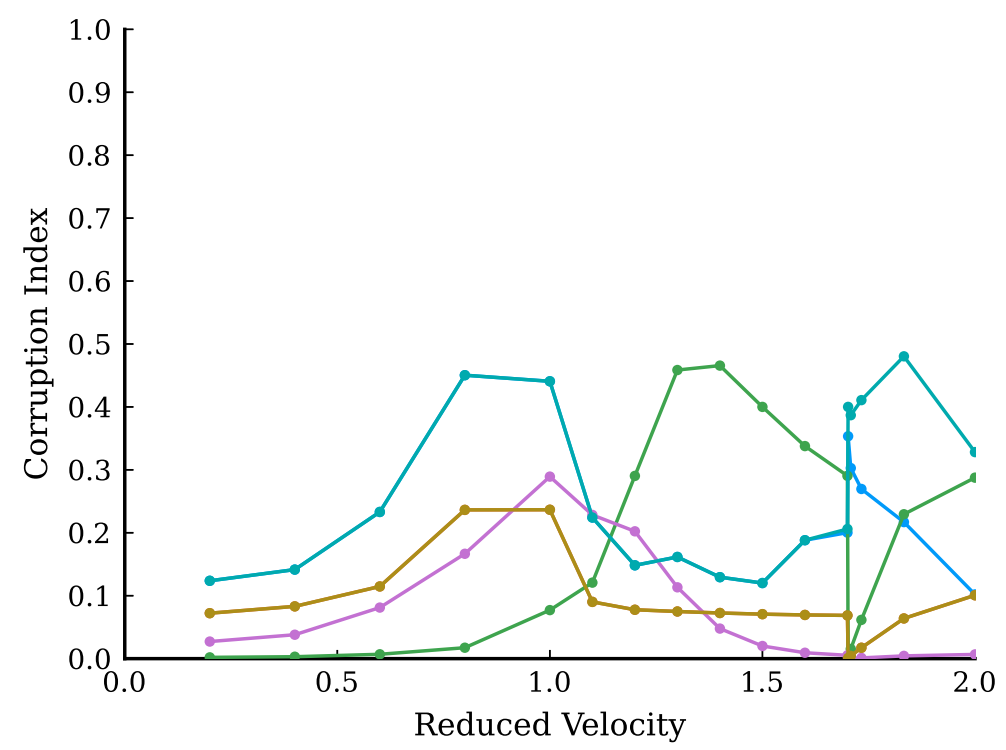

Fig. 7 New mode tracking method corruption indices for each mode association of the 2D aeroelastic system

Table 1 Highly Flexible Slender Wing Properties

\begin{tabular}{lr}
\hline \hline Property & Value \\
\hline Span & $16 \mathrm{~m}$ \\
Chord & $1 \mathrm{~m}$ \\
Spanwise ref. axis location (from 1.e.) & $50 \%$ of chord \\
Center of gravity (from 1.e.) & $50 \%$ of chord \\
Flat bending rigidity & $2 \times 10^{4} \mathrm{~N} \mathrm{~m}^{2}$ \\
Chord bending rigidity & $4 \times 10^{6} \mathrm{~N} \mathrm{~m}^{2}$ \\
Torsional rigidity & $1 \times 10^{4} \mathrm{~N} \mathrm{~m}^{2}$ \\
Mass per unit span & $0.75 \mathrm{~kg} / \mathrm{m}$ \\
Rotational inertia per unit span & $0.1 \mathrm{~kg} \mathrm{~m}$ \\
\hline
\end{tabular}

The corruption index associated with each mode association in fig. 6 is shown in fig. 7. The corruption indices for all velocity increments remain below the tolerance of 0.5 , indicating that the mode associations are likely correct. The low corruption indices shown in fig. 7 also confirm that our mode tracking method is able to reduce corruption indices to an arbitrary level.

\section{B. Nonlinear 3D Aeroelastic Analysis}

For this case, we test the performance of our new mode tracking method when modeling the stability of a highly flexible slender wing with properties as specified in table 1. For the beam's structure, we use the geometrically exact beam model described previously with eight beam elements. To calculate the distributed aerodynamic loads on each beam element, we use Peter's finite state model with six aerodynamic state variables for each section. We assume the wing is cantilevered at a $2^{\circ}$ angle of attack and subjected to a freestream velocity ranging from $1 \mathrm{~m} / \mathrm{s}$ to $35 \mathrm{~m} / \mathrm{s}$ with a default velocity increment of $1 \mathrm{~m} / \mathrm{s}$. We assume the air density for the analysis is $0.088 \mathrm{~kg} / \mathrm{m}$. These properties match the properties given for a similar analysis performed by Hang et al.[21]

The frequency and damping ratio, with eigenvalue sorting applied using C-CORC, is plotted in fig. 8 . The corresponding corruption indices for the aeroelastic mode correlations are plotted in fig. 9 . Some mode-switching may is observed in the post-flutter region (at velocities greater than $22 \mathrm{~m} / \mathrm{s}$ ) as evidenced by the rapidly changing plotted frequency and damping. The presence of mode switching is also evidenced by the high corruption index values in 

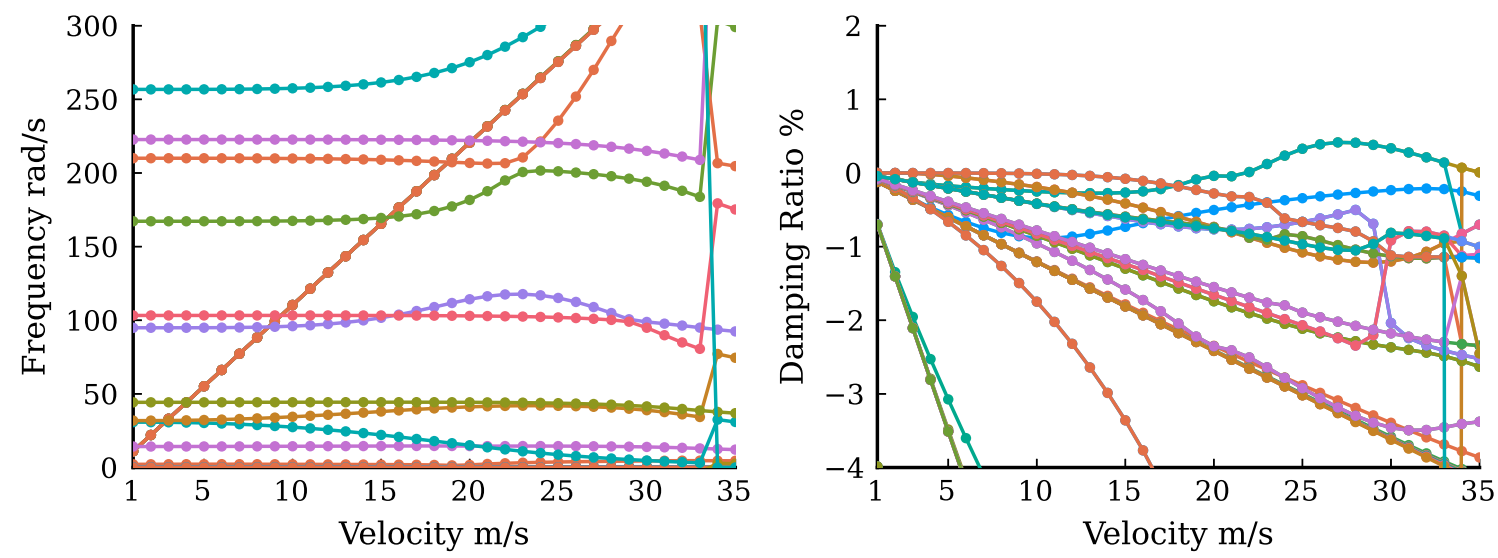

Fig. 8 Frequency and damping of the 3D aeroelastic system with sorting using C-CORC

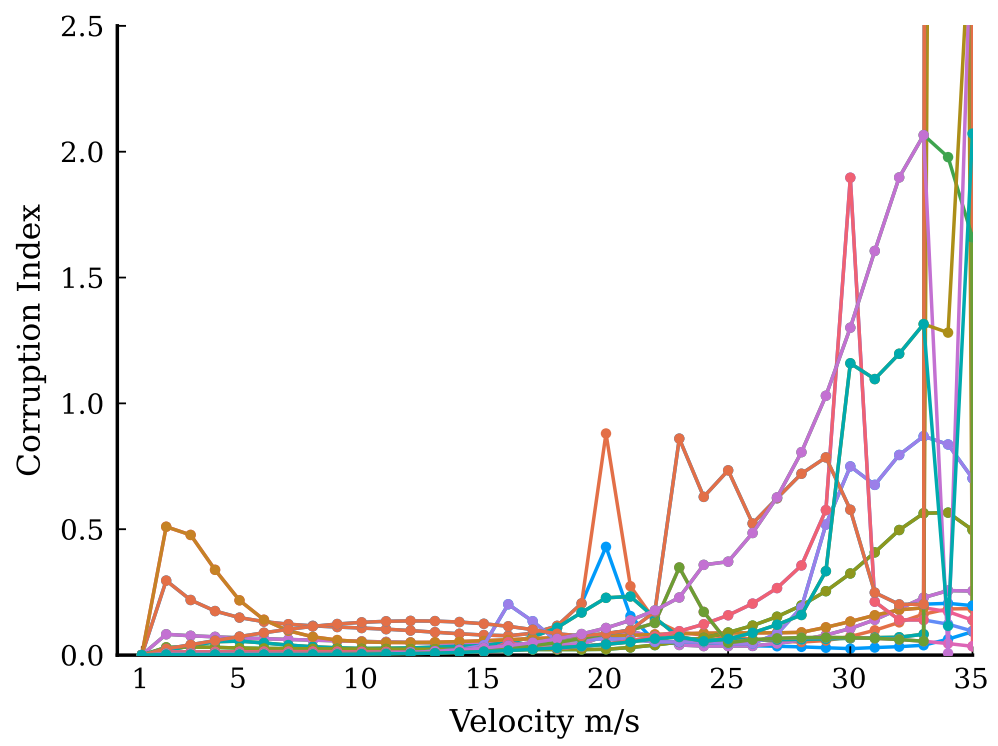

Fig. 9 C-CORC corruption indices for each aeroelastic mode association for the 3D aeroelastic system 

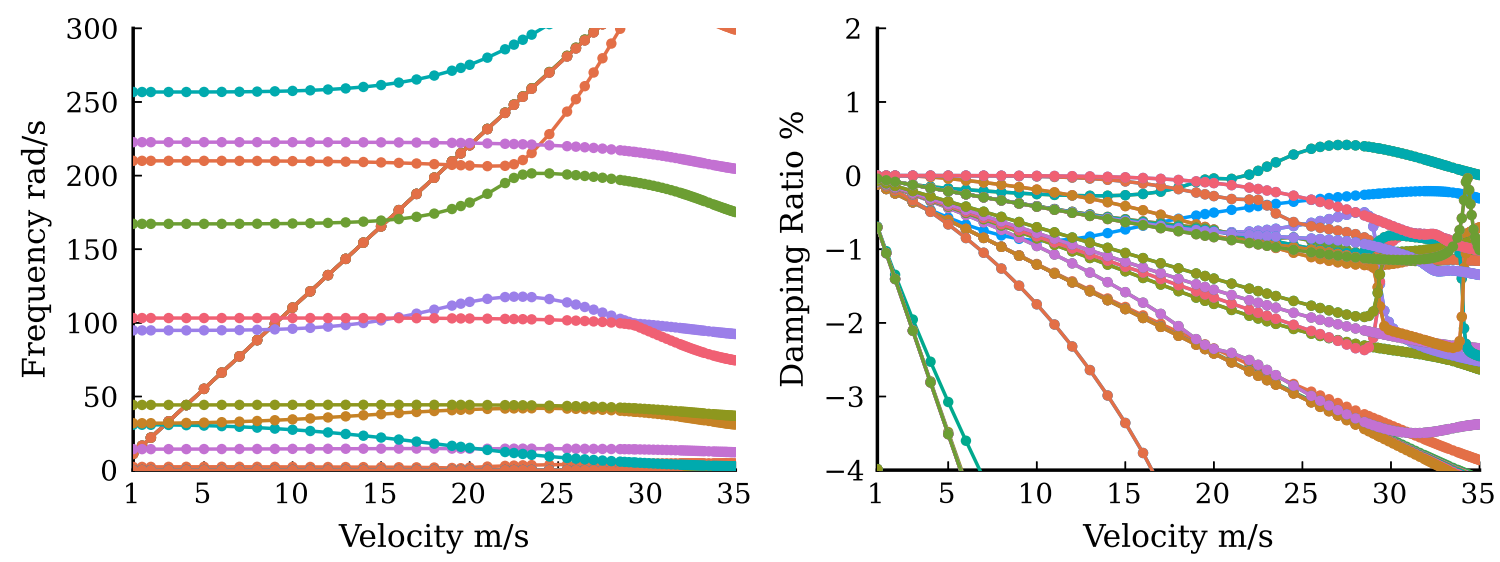

Fig. 10 Frequency and damping of the 3D aeroelastic system with sorting using the new mode tracking method presented in this paper

the same post-flutter region, with many corruption indices exceeding one. On the other hand, low corruption indices at lower velocities suggest that larger velocity increments could have been used at lower velocities while maintaining correct mode associations.

Figure 10 and fig. 11 show the result of applying the new mode tracking technique presented in this paper, with a corruption index tolerance of 0.5 and simple backtracking logic that halves the step size if the corruption index tolerance is exceeded. Note that the corruption index tolerance in this case is applied only to the aeroelastic modes, as identified at a velocity of $1 \mathrm{~m} / \mathrm{s}$. In addition to fixing all issues associated with mode switching, our mode tracking method confirms that some of the rapidly changing frequency and damping values in the post-flutter region are in fact a characteristic of the system, and not just an artifact of the mode tracking step size.

\section{Conclusions}

In this paper, a new mode tracking method was presented which incorporates backtracking logic in order to generate mode correlations with an arbitrarily high degree of confidence. This mode tracking method was demonstrated on both linear two-dimensional and nonlinear three-dimensional aeroelastic systems, but is applicable to all systems whose governing equations may be expressed as a set of first-order ordinary differential equations. By maintaining an extremely high degree of confidence in calculated mode correlations, this mode tracking method is able to be used in scenarios in which obtaining correct mode associations are critical, such as when constructing mode specific flutter constraints and/or objectives for gradient-based optimization frameworks.

The new mode tracking method presented in this paper is based on the complex cross-orthogonality check method (C-CORC) presented by Eldred et al.[20], but uses an adaptive, rather than fixed, step size based on the measured accuracy of the mode correlations. In this new mode tracking method, mode correlations are generated based on the creation of a correlation matrix, generated using the left and right eigenvectors and the system mass matrix, if applicable. Then the corruption index, a measure of the likelihood of a false mode association, is determined based on entries in the correlation matrix for each mode association. Finally, the proposed step size is reduced if the corruption index exceeds a user-specified tolerance, which may be arbitrarily small. Since the mode tracking method presented in this paper is able to prescribe an arbitrarily small maximum corruption index tolerance, it is able to generate mode correlations with an arbitrarily high degree of confidence.

For both the linear two-dimensional and nonlinear three-dimensional aeroelastic analyses considered in this paper, C-CORC was able to eliminate most occurrences of mode switching, whereas the new mode tracking method was able to eliminate all occurrences of mode switching. The new mode tracking method was also found to be capable of identifying and tracking rapidly changing modes, even when used with a large default step sizes (relative to the gradient of the changing modes). The insensitivity of the new mode tracking to the default step size allows it to be accurate even in contexts in which the sensitivity of the aeroelastic modes to a step size change is unknown, which makes it extremely useful when applied to track modes during aeroelastic analyses and multidisciplinary design optimizations. 


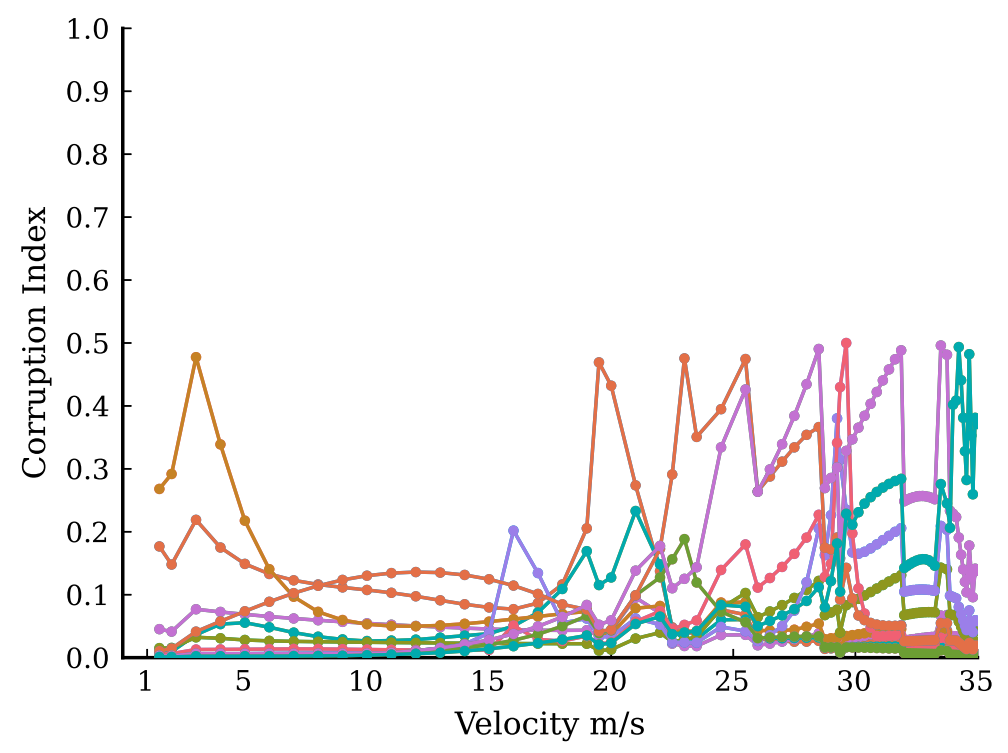

Fig. 11 New mode tracking method corruption indices for each mode association of the 3D aeroelastic system

\section{Acknowledgments}

The authors gratefully acknowledge support from the Utah NASA Space Grant Consortium.

\section{References}

[1] Garrigues, E., “A Review of Industrial Aeroelasticity Practices at Dassault Aviation for Military Aircraft and Business Jets," Aerospace Lab, , No. 14, 2018, pp. 1-34. https://doi.org/10.12762/2018.AL14-09.

[2] Kenway, G. K. W., and Martins, J. R. R. A., "Multipoint High-Fidelity Aerostructural Optimization of a Transport Aircraft Configuration," Journal of Aircraft, Vol. 51, No. 1, 2014, pp. 144-160. https://doi.org/10.2514/1.c032150

[3] Variyar, A., Economon, T. D., and Alonso, J. J., "Design and Optimization of Unconventional Aircraft Configurations with Aeroelastic Constraints," AIAA SciTech Forum, American Institute of Aeronautics and Astronautics, 2017. //doi.org/10.2514/6.2017-0463

[4] Yu, Y., Lyu, Z., Xu, Z., and Martins, J. R., "On the influence of optimization algorithm and initial design on wing aerodynamic shape optimization,” Aerospace Science and Technology, Vol. 75, 2018, pp. 183 - 199. https://doi.org/10.1016/j.ast.2018.01.016

[5] Haftka, R. T., "Automated Procedure for Design of Wing Structures to Satisfy Strength and Flutter Requirements," Tech. Rep. TN-D-7264, NASA Langley Research Center, 1973.

[6] Langthjem, M., and Sugiyama, Y., "Optimum Shape Design against Flutter of a Cantilevered Column with an End-Mass of Finite Size subjected to a Non-Conservative Load," Journal of Sound and Vibration, Vol. 226, No. 1, 1999, pp. 1-23. https://doi.org/10.1006/jsvi.1999.2211

[7] Odaka, Y., and Furuya, H., "Robust structural optimization of plate wing corresponding to bifurcation in higher mode flutter," Structural and Multidisciplinary Optimization, Vol. 30, No. 6, 2005, pp. 437-446. https://doi.org/10.1007/s00158-005-0538-9

[8] Hajela, P., “A root locus-based flutter synthesis procedure,” Journal of Aircraft, Vol. 20, No. 12, 1983, pp. $1021-1027$. https://doi.org/10.2514/3.48206

[9] Ringertz, U. T., “On structural optimization with aeroelasticity constraints," Structural optimization, Vol. 8, No. 1, 1994, pp. 16-23. https://doi.org/10.1007/BF01742928

[10] Stanford, B., Wieseman, C. D., and Jutte, C., "Aeroelastic Tailoring of Transport Wings Including Transonic Flutter Constraints," AIAA SciTech Forum, American Institute of Aeronautics and Astronautics, 2015. https://doi.org/10.2514/6.2015-1127

[11] Stanford, B. K., "Role of Unsteady Aerodynamics During Aeroelastic Optimization," AIAA Journal, Vol. 53, No. 12, 2015 , pp. 3826-3831. https://doi.org/10.2514/1.J054314 
[12] Kreisselmeier, G., and Steinhauser, R., "Systematic Control Design by Optimizing a Vector Performance Index," IFAC Proceedings Volumes, Vol. 12, No. 7, 1979, pp. 113-117. https://doi.org/10.1016/s1474-6670(17)65584-8

[13] Wrenn, G. A., "An indirect method for numerical optimization using the Kreisselmeir-Steinhauser function," Tech. Rep. CR-4220, NASA Langley Research Center, Hampton, VA, 1989.

[14] Poon, N. M. K., and Martins, J. R. R. A., "An adaptive approach to constraint aggregation using adjoint sensitivity analysis," Structural and Multidisciplinary Optimization, Vol. 34, No. 1, 2007, pp. 61-73. https://doi.org/10.1007/s00158-006-0061-7

[15] McDonnell, T., and Ning, A., "Gradient-Based Optimization of Solar-Regenerative High-Altitude Long-Endurance Aircraft," Journal of Aircraft, Vol. 57, No. 6, 2020, pp. 1189-1201. https://doi.org/10.2514/1.C035566

[16] Lupp, C. A., and Cesnik, C. E., "A Gradient-Based Flutter Constraint Including Geometrically Nonlinear Deformations," AIAA SciTech Forum, American Institute of Aeronautics and Astronautics, 2019. https://doi.org/10.2514/6.2019-1212.

[17] Desmarais, R. N., and Bennett, R. M., "An Automated Procedure for Computing Flutter Eigenvalues," Journal of Aircraft, Vol. 11, No. 2, 1974, pp. 75-80. https://doi.org/10.2514/3.60326

[18] Chen, P. C., "Damping Perturbation Method for Flutter Solution: The g-Method," AIAA Journal, Vol. 38, No. 9, 2000, pp. 1519-1524. https://doi.org/10.2514/2.1171.

[19] van Zyl, L. H., "Use of eigenvectors in the solution of the flutter equation," Journal of Aircraft, Vol. 30, No. 4, 1993, pp. 553-554. https://doi.org/10.2514/3.46380

[20] Eldred, M. S., Venkayya, V. B., and Anderson, W. J., "New mode tracking methods in aeroelastic analysis," AIAA Journal, Vol. 33, No. 7, 1995, pp. 1292-1299. https://doi.org/10.2514/3.12552

[21] Hang, X., Fei, Q., and Su, W., "On Tracking Aeroelastic Modes in Stability Analysis Using Left and Right Eigenvectors,” AIAA Journal, Vol. 57, No. 10, 2019, pp. 4447-4457. https://doi.org/10.2514/1.j057297

[22] Jones, R. T., “The Unsteady Lift of a Wing of Finite Aspect Ratio,” Tech. Rep. TR-681, NACA, Washington, D.C., 1940.

[23] Peters, D. A., Karunamoorthy, S., and Cao, W.-M., "Finite state induced flow models. I - Two-dimensional thin airfoil," Journal of Aircraft, Vol. 32, No. 2, 1995, pp. 313-322. https://doi.org/10.2514/3.46718

[24] Yu, W., and Blair, M., "GEBT: A general-purpose nonlinear analysis tool for composite beams," Composite Structures, Vol. 94, No. 9, 2012, pp. 2677-2689. https://doi.org/10.1016/j.compstruct.2012.04.007

[25] Wang, Q., and Yu, W., "Geometrically nonlinear analysis of composite beams using Wiener-Milenković parameters,” Journal of Renewable and Sustainable Energy, Vol. 9, No. 3, 2017, p. 033306. https://doi.org/10.1063/1.4985091. 